\title{
A Study of the Experience of German Dental Practitioners with Patients on Bisphosphonates or Anticoagulants
}

\author{
Mihaela Furnadzhieva, \\ Zahnarzt Stuttgart, Germany
}

\begin{abstract}
Introduction: The well-structured and functioning dental care system in Germany is a generally recognized fact. The extensive German experience in the field of dental medicine has yielded regulations providing guidance on work with vulnerable social groups. Patients planned to undergo bisphosphonate or anticoagulant therapy need dental sanitation prior to treatment according to a predefined treatment scheme. In case surgery is required during drug intake, specific rules are to be followed.

Aim: A study of the experience of German dental practitioners with patients on bisphosphonates or anticoagulants at outpatient medical facilities

Material and Methods: The clinical study was performed with 40 patients receiving bisphosphonate or anticoagulants treatment and examined in Germany between 2015 and 2017. Also, it included a total of 150 dental practitioners working in Baden-Württemberg, Federal Republic of Germany.

Conclusion: Dental care for patients on bisphosphonates or anticoagulants in Germany is organized by the government and can be delivered at any dental practice.
\end{abstract}

Keywords: bisphosphonates, anticoagulants, Germany, sanitation and clinical protocol

\section{Introduction}

Nearly all patients coming to a dentist's office receive some kind of medication. This need is to be duly documented in their medical records and taken into account when administering dental care. The dental 
practitioner is expected to be aware of the risks related to the intake of some drugs and apply the necessary preventive measures and control to avoid complications. Bisphosphonates and anticoagulants are examples of such medications. $(1,2,3,4)$

\section{Bisphosphonates}

Bisphosphonates are used in the treatment of various benign conditions and malignancies involving bone metabolic disorders and hypercalcaemia, e.g. multiple myeloma, and other bone metastatic cancers; osteoporosis; Paget's disease.(5)

Patients planned to undergo this type of therapy need dental sanitation prior to treatment, according to a predefined treatment scheme. In case surgery is required during drug intake, specific rules are to be followed. A possible complication is bisphosphonate-related osteonecrosis of the jaw (BRONJ) manifesting as exposed, nonvital bone that does not recover over 8 weeks after current or past bisphosphonate therapy $(6,7,8,9,10,11,12)$

The mains steps included in the Germany clinical protocol for dental sanitation and care in patients receiving or planned to receive bisphosphonate therapy are as follows: $(13,14,15)$

1. Primary prevention prior to bisphosphonate therapy

\section{Dental examination}

Consulting and briefing the patient on the type of drugs used in bisphosphonate therapy, their side effects, and the patient's line of conduct in case of discomfort

Dental sanitation - to be performed 14 to 21 days prior to the beginning of therapy

- optimizing personal and professional oral hygiene - patient training and motivation to maintain a high level of oral hygiene

- conservative and endodontic treatment

- periodontal treatment

- focus sanitation

- surgical sanitation of all teeth of poor prognosis, management of bone defects

- adjustment of removable partial and total prostheses to prevent decubital damage of the oral mucosa and jaw bones

Follow up every 3,6 , and 12 months

2. Dental care if needed during bisphosphonate therapy

Conservative treatment - sanitation (filling) of caries lesions having occurred during bisphosphonate therapy

Prosthetic treatment can be performed during bisphosphonate therapy using fixed prosthodontics or removable dentures to restore the wholeness of teeth rows in order to prevent trauma and ensure 
normal masticatory function.

Surgery in dental emergency - tooth extraction, management of bone defects, treatment of oral mucosa lesions

- Similar interventions can be undertaken 14 days after the last intake of the drug at the earliest and after preventive administration of antibiotics.

- Postoperatively, medication is to be discontinued for another 14 days.

- Extractions need to be as atraumatic as possible; idem for the needles and sutures used; a flap is used to shorten the recovery period.

3. Secondary prevention by supportive treatment with: Chlorhexidindiglukonat 0,12 percent (1-2 percent), Wasserstoffperoxid-Lösung 1 percent and professional oral hygiene with supragingival scaling.

Patients receiving anticoagulant therapy are at no lesser risk. Dental care delivered to such patients also requires that the dental practitioner be prepared to follow specific rules.

\section{Anticoagulants}

Anticoagulants are drugs that inhibit blood clotting mechanisms, directly or indirectly. Anticoagulants are classified according to their mechanism of action intro the following main groups:

- Indirect thrombin inhibitors: heparin and low molecular weight heparins

- Direct factor Xa inhibitors

- Direct thrombin inhibitors

- Coumarin anticoagulants.

The mains steps included in the Germany clinical protocol for dental sanitation and care in patients receiving or planned to receive anticoagulant therapy are as follows: $(16,17)$

- The duration of therapy depends on each individual case and can last for years or even lifelong in some patients.

- During therapy prothrombin time (PT) and international normalized ratio (INR) need to be monitored periodically.

- Surgery in dental emergency - tooth extraction, management of bone defects, treatment of oral mucosa lesions. Extractions need to be as atraumatic as possible; idem for the needles and sutures used; a flap is used to shorten the recovery period. Drugs shortening blood clotting time are used and compressive dressings are applied on the surgical site.

- Several prevention schemes exist to discontinue or replace the usual treatment. These are discussed with the treating physician based on the number and type of surgical interventions to be performed and the duration of action of the drug involved.

Bleeding is the most frequent side effect of anticoagulant therapy. If patients receiving anticoagulants need dental surgery, their therapy is temporarily discontinued, with the duration of pause varying from one drug to another. The decision is made in close collaboration with the treating physician. 


\section{Aim:}

A study of the experience of German dental practitioners with patients on bisphosphonates or anticoagulants at outpatient medical facilities.

\section{Material and Methods:}

The clinical study included 40 patients aged between 30 and 80 years, of whom 20 receiving anticoagulants and 20 receiving bisphosphonates, all examined at outpatient dental clinics in BadenWürttemberg, Federal Republic of Germany, between 2015 and 2017 and diagnosed according to the German standards. Results were classified into the following groups: number of preventive examinations per year after the beginning of therapy and number of extractions during therapy.

The study was also performed with a total of 150 dental practitioners at outpatient medical facilities in Baden-Württemberg, Federal Republic of Germany. They were asked to fill the following questionnaire between 2015 and 2017:

1. Have you performed dental care in patients receiving bisphosphonates or blood thinners?

Yes $\square \quad$ No

2. Do you keep in touch with the general practitioner treating of this group of patients?

$\square \quad$ Yes $\square \quad$ No

3. Do you feel sufficiently informed about the way to work with this group of patients?

$\square \quad$ Yes $\square \quad$ No

4. Do you feel sufficiently informed about the side effects and risks involved in the intake of these drugs?

$\square \quad$ Yes $\square \quad$ No

5. Do you feel sufficiently informed about the preventive measures to undertake prior to dental care in this group of patients?

$\square \quad$ Yes $\square \quad$ No

6. Were there complications after dental care in this group of patients?

$\square \quad$ Yes $\square \quad$ No

7. What kind of difficulties do you encounter most when working with this group of patients?

8. Please make recommendations about how the work with this group of patients can be improved. 


\section{Results and discussion:}

\section{Fig.1 Number of preventive examinations per year after the beginning of bisphosphonate therapy}

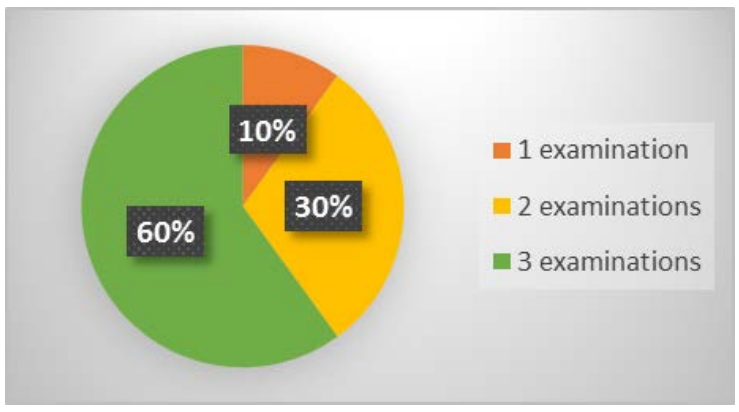

The main group of 20 patients on bisphosphonates received a total number of 50 preventive examinations per year after the beginning of treatment as follows: 2 patients (10 percent) had 1 examination; 6 patients (30 percent) had two examinations, and 12 patients (60 percent) had three examinations. Notably, all patients from this study group (100 percent) underwent preventive examinations, some had two (30percent) or three (60percent).

\section{Fig.2 Number of extractions during therapy}

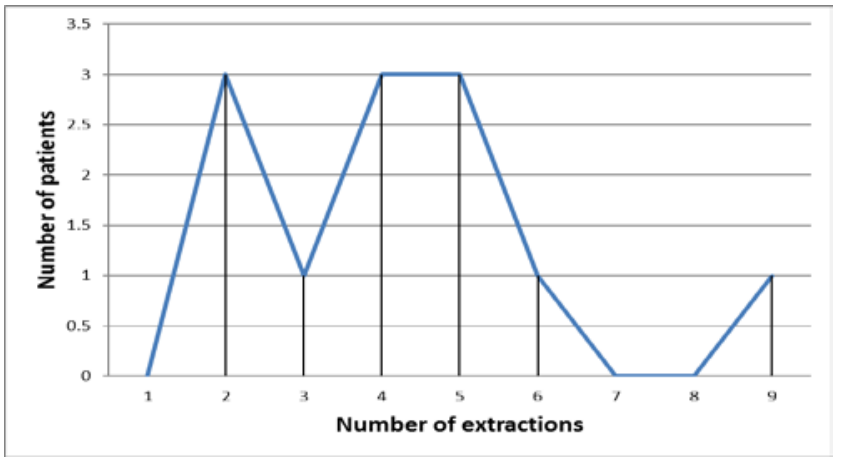

Fig.2 shows the number of extracted teeth (47) in the group of 20 patients treated during bisphosphonate therapy. Analysis reveals that three of the patients had 2 teeth extracted, one had 3 teeth extracted, three had 4 teeth extracted, three had 5 teeth extracted, one had 6 teeth extracted and one had 9 teeth extracted.

\section{Fig.3 Number of preventive examinations per year after the beginning of anticoagulant therapy}

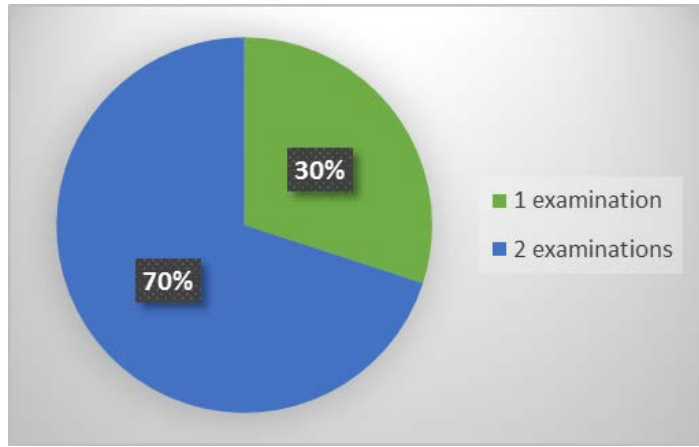

The main group of 20 patients on anticoagulants a total number of 34 preventive examinations per year after the beginning of treatment as follows: 6 patients (30 percent) had one examination and 14 (70 percent) had two examinations. Notably, all cancer patients having participated in the study (100 percent) had a preventive examination (100 percent) and most (70percent) had two. 


\section{Fig.3 Number of extractions during therapy}

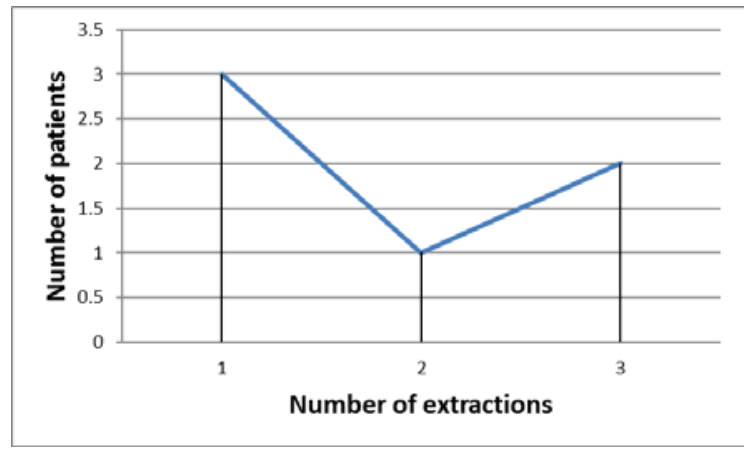

Fig. 3 shows the number of extracted teeth (11) in the group of 20 patients treated during anticoagulant therapy. Analysis reveals that three patients had 1 tooth extracted, one had 2 teeth extracted, and two had 3 teeth extracted.

Fig.4 Answers to question № 1: Have you performed dental care in patients receiving bisphosphonates or blood thinners?

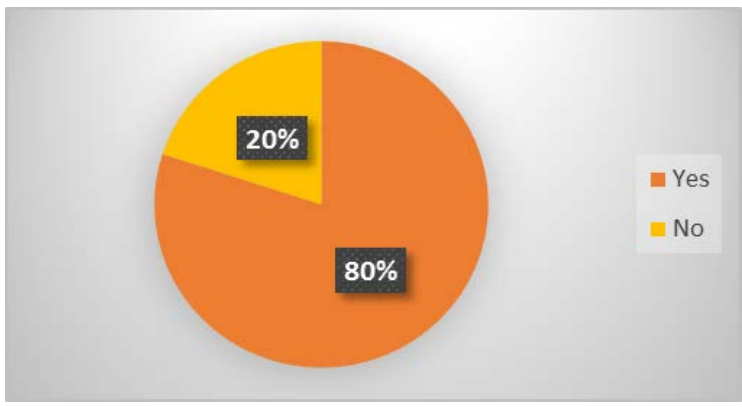

Question № 1 was answered by all 150 dental practitioners, of whom 80 percent had treated patients receiving bisphosphonate therapy and 20 percent had not.

Fig.5 Answers to question № 2: Do you keep in touch with the general practitioner treating of this group of patients?

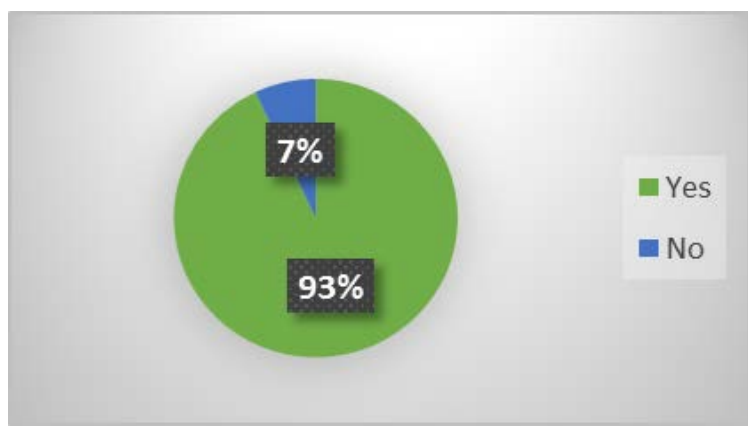

Question № 2 was answered by 123 dental practitioners, of whom 93 percent do keep in touch with the general practitioner treating this group of patients, and 7 percent do not. 
Fig.6 Answers to question № 3: Do you feel sufficiently informed about the way to work with this group of patients?

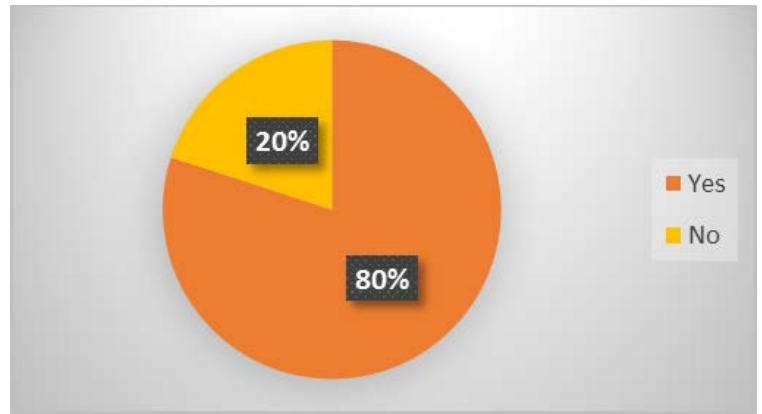

Question № 3 was answered by 123 dental practitioners, of whom 80 percent feel sufficiently informed about the way to work with this group of patients.

Fig.7 Answers to question № 4: Do you feel sufficiently informed about the side effects and risks involved in the intake of these drugs?

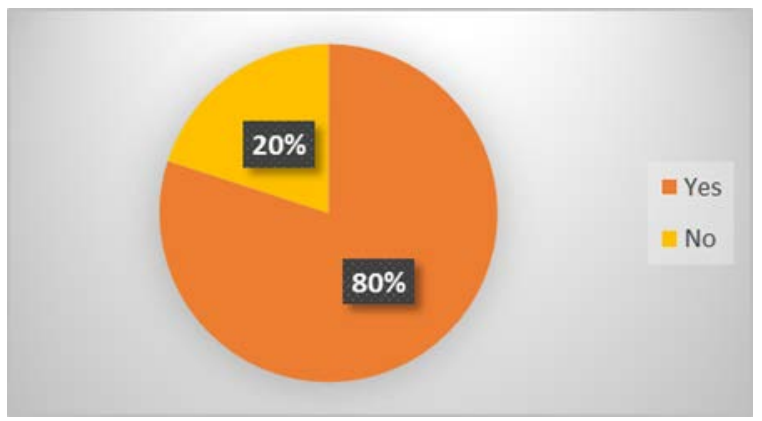

Question № 4 was answered by 123 dental practitioners, 80 percent feel sufficiently informed about the side effects and risks involved in the intake of these drugs.

Fig.8 Answers to question № 5: Do you feel sufficiently informed about the preventive measures to undertake prior to dental care in this group of patients?

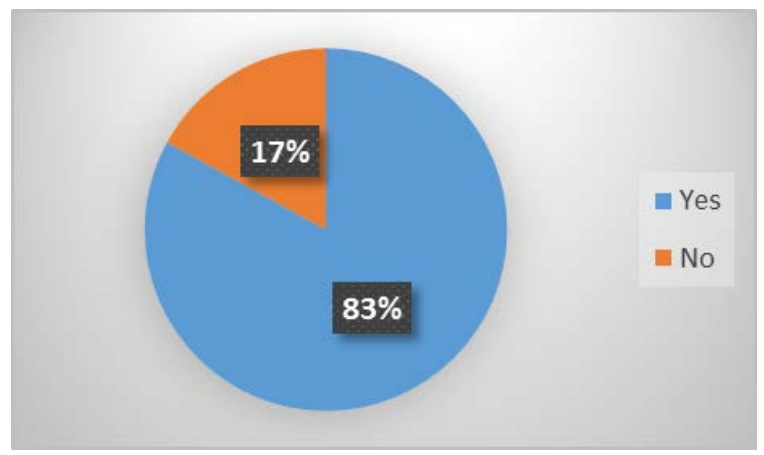

Question № 5 was answered by 123 dental practitioners, of whom 83 percent feel sufficiently informed about the preventive measures to undertake prior to dental care in this group of patients. 


\section{Fig.9 Answers to question № 6 : Were there complications after dental care in this group of} patients?

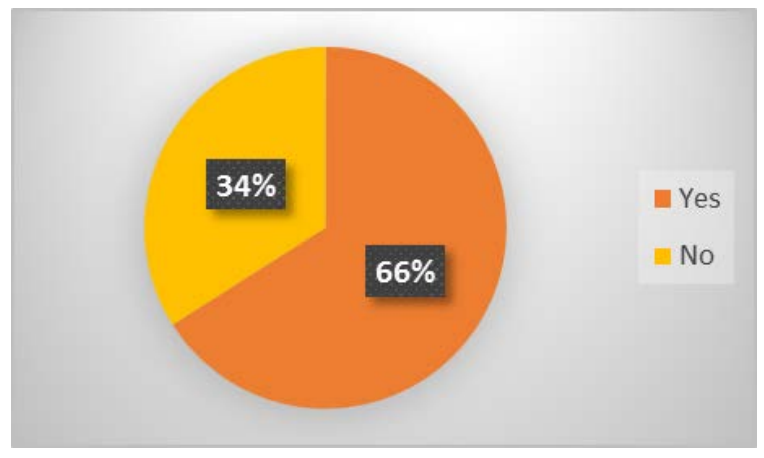

Question № 6 was answered by 123 dental practitioners, of whom 66 percent reported lack of complications after dental treatment administered to these patients.

\section{Answers to question № 7: What kind of difficulties do you encounter most when working with this} group of patients?

Three difficulties prevailed: bleeding, uncertainty in choosing an antibiotic, and uncertainty in choosing a treatment plan.

Answers to question № 8: Please make recommendations about how the work with this group of patients can be improved.

The following recommendations prevail:

- More information to be provided to both patients and therapists

- Information courses for dental practitioners on working with patients receiving bisphosphonate therapy

- Better briefing of the patient by the general practitioner.

\section{Conclusion}

If patients receiving these drugs need dental surgery, therapy is temporarily discontinued, with the duration of pause varying from one drug to another. The decision is always made in close collaboration with the treating physician.

The regularity of surveys conducted among German dental practitioners in Baden-Württemberg, Federal Republic of Germany reveal a high level of knowledge and professional commitment. This mode of work is the result of the ongoing control exerted by health insurance funds and can be put into practice in our country as well. 


\section{References}

1. Deliversky, J., M. Yaneva-Deliverska, M. Lyapina, A. Kisselova. European and international standards on medical devices for dentistry. J of IMAB, 2015 Jan-Mar;21(1):713-717.

2. Yaneva-Deliverska, M., J. Deliversky, M. Lyapina. Biocompatibility of medical devices - legal regulations in the European Union. J of IMAB, 2015 Jan-Mar;21(1):705-708.

3. Deliversky, J. Information Security in Medical Institutions. Measures for Improvement, Burgas Free University, New Idea in Education, 2016, vol. 2, p. 77-82 [in Bulgarian]

4. Yaneva-Deliverska M., Legal aspects and legal regulation of dental alergology in oral medicine, Alergology in oral medicine: principles ans practices (textbook under the review of A. Kisselova Yaneva and B. Petrunov) [In Bulgarian]

5. Di Fede O, Bedogni A, Giancola F, Saia G, Bettini G. BRONJ in patients with rheumatoid arthritis: a multicenter case series. Oral Dis. 2016;22(6):543-8.

6. Paulßen F, Paashaus M, Hammacher A. Primärverdacht BRONJ zeigt sich als Metastase. ZM. 2016;16.

7. Kwon YD, Lee CY, Hong SO, Lee YA, Ohe JY, Kim DY. Bisphosphonate related osteonecrosis of the jaws (BRONJ) in osteoporotic males. Springerplus. 2016;5(1):1468.

8. Comas-Calonge A, Figueiredo R, Gay-Escoda C. Surgical treatment vs. conservative treatment in intravenous bisphosphonate-related osteonecrosis of the jaws. Systematic review. J Clin Exp Dent. 2017 Feb 1;9(2):e302-e307.

9. Jakiel J, Rahnama M, Szczerba-Gwóźdź J. Treatment of bisphosphonate-related osteonecrosis of the jaws - a report of seven cases. Contemp Oncol (Pozn). 2016;20(6):486-490.

10. Janus JR, Jackson RS, Lees KA, Voss SG, Wilson ZC. Human Adipose-Derived Mesenchymal Stem Cells for Osseous Rehabilitation of Induced Osteoradionecrosis. Otolaryngol Head Neck Surg. 2017:194599816688647.

11. Niewald M, Mang K, Barbie O et al. Dental status, dental treatment procedures and radiotherapy as risk factors for infected osteoradionecrosis (IORN) in patients with oral cancer - a comparison of two 10 years' observation periods. Springerplus 2014;3:263.

12. Rollason V, Laverrière A, MacDonald LC, Walsh $T$, Tramèr MR, Vogt-Ferrier NB. Interventions for treating bisphosphonate-related osteonecrosis of the jaw (BRONJ). Cochrane Database Syst Rev. 2016;2:CD008455.

13. Lain R1,2, Ajwani S1,2. Minor post-extraction complications other than BRONJ in older patients on oral bisphosphonates - a retrospective study. Gerodontology. 2016. doi: 10.1111/ger.12239.

14. S3-Leitlinie: Bisphosphonat- assoziierte Kiefernekrose (BP-ONJ) und andere Medikamentenassoziierte Kiefernekrosen. 2012.

15. Vescovi P, Nammour S. Bisphosphonate-Related Osteonecrosis of the Jaw (BRONJ) therapy. A critical review. Minerva Stomatol. 2010;59:181-203,204-13.

16. Schwenzer N, Ehrenfeld M Zahn-Mund-Kiefer-Heilkunde. fourth edition. Publisher: Thieme. 2008.

17. Schwenzer N, Ehrenfeld M. Zahn-Mund-Kiefer-Heilkunde: Zahnärztliche Chirurgie. fourth edition. Publisher: Thieme 2010.

\section{Corresponding author:}

Mihaela Furnadzhieva

Dentist Stuttgart, Germany

Email: mihaelafurnadzhieva@gmail.com 